\title{
Supporting Information for Time-Resolved
}

\section{Photoionization Detection of a Single $\mathrm{Er}^{3+}$ Ion in Silicon}

Guangchong Hu, ${ }^{\dagger}$ Gabriele G. de Boo, ${ }^{\dagger}$ Brett Cameron Johnson,,${ }^{\ddagger}$ Jeffrey Colin McCallum, ${ }^{\ddagger}$ Matthew J. Sellars, ${ }^{\S}$ Chunming Yin $,{ }^{*} \dagger, \|$ and Sven Rogge ${ }^{*, \dagger}$

$\dagger$ Centre of Excellence for Quantum Computation and Communication Technology, School of Physics, University of New South Wales, NSW 2052, Australia

$\ddagger$ Centre of Excellence for Quantum Computation and Communication Technology, School of Physics, University of Melbourne, Victoria 3010, Australia

ICentre of Excellence for Quantum Computation and Communication Technology, School of Engineering, RMIT University, Victoria 3001, Australia

$\S$ Centre of Excellence for Quantum Computation and Communication Technology, Research School of Physics and Engineering, Australian National University, ACT 0200, Australia $\|$ CAS Key Laboratory of Microscale Magnetic Resonance, School of Physical Sciences and CAS Center for Excellence in Quantum Information and Quantum Physics, University of Science and Technology of China, Hefei 230026, China

E-mail: chunming@ustc.edu.cn; s.rogge@unsw.edu.au

\section{Methods}

The silicon nano-transistor used in this study was a fin field-effect transistor (FinFET), that had a single crystalline silicon nanowire channel with a width and length of $35 \mathrm{~nm}$ 
$\times 100 \mathrm{~nm}$, respectively. The erbium isotope ${ }^{170} \mathrm{Er}$ with zero nuclear spin, and O, were implanted through a $100 \mathrm{~nm}$ thick gate layer into the channel. There was an estimated 30 $\mathrm{Er}^{3+}$ ions in the channel, and the expected Er:O ratio was 1:6. The device was then annealed at $700{ }^{\circ} \mathrm{C}$ in $\mathrm{N}_{2}$ for 10 minutes to reduce damage originating from the implantation process and to optically activate the Er centres.

The Er-doped FinFET was investigated at $4.2 \mathrm{~K}$ in an helium bath cryostat. The Er spectral signal was measured on either of the two Zeeman branches at low magnetic fields (up to $30 \mathrm{mT}$ ) or the degenerate state at zero field. The sample was illuminated with a single-mode fibre (Corning SMF-28e) and the tip of the fibre was aligned with the channel of the transistor under an optical microscope. The laser spot diameter on the device was approximately $500 \mu \mathrm{m}$.

A tunable laser was used to resonantly excite the $\mathrm{Er}^{3+}{ }^{4} \mathrm{I}_{15 / 2}{ }^{-4} \mathrm{I}_{13 / 2}$ optical transition. For the pulsed experiments, an electro-optic modulator (EOM) was used to create two frequency and amplitude tunable side bands controlled by RF frequency and power, and DC voltage was set on the EOM to minimise the optical carrier band. An acousto-optic modulator (AOM) was also used for pulsing, and the EOM and AOM together provided a total extinction ratio of $70 \mathrm{~dB}$. The laser power specified in this study corresponds to the total optical power before the fibre port on top of the cryostat.

The FinFET was biased slightly below its threshold voltage, where a quantum dot forms at the corners of the channel due to geometric enhancement of the electric field ${ }^{1}$. As a result the FinFET works as a charge-sensitive single electron transistor (SET) with current through the quantum dot arising from single electron tunnelling ${ }^{2}$. The device was then illuminated with the tunable laser. When the laser frequency was resonant with the optical transition of an $\mathrm{Er}^{3+}$ ion, the $\mathrm{Er}^{3+}$ ion was excited from the ${ }^{4} \mathrm{I}_{15 / 2}$ state to the ${ }^{4} \mathrm{I}_{13 / 2}$ state, and then relaxed back to the ${ }^{4} \mathrm{I}_{15 / 2}$ via radiative or non-radiative processes. One of the relaxation processes can ionise a nearby charge trap and change the electrochemical potential of the SET, leading to a change in the tunnelling current ${ }^{3}$. 


\section{Additional Er spectra}

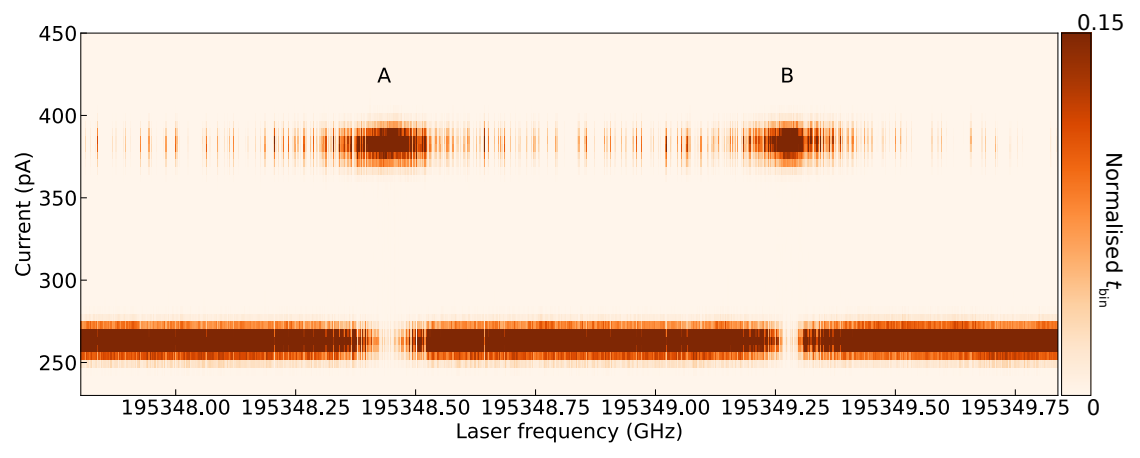

Figure S1: The full spectrum of two Zeeman doublet transitions of the single $\mathrm{Er}^{3+}$ ion at $20 \mathrm{mT}$. The region around Peak ' $\mathrm{B}$ ' is presented as Fig. 1(a) in the main manuscript. This devices is labelled as Er-1 in the Supporting Information.
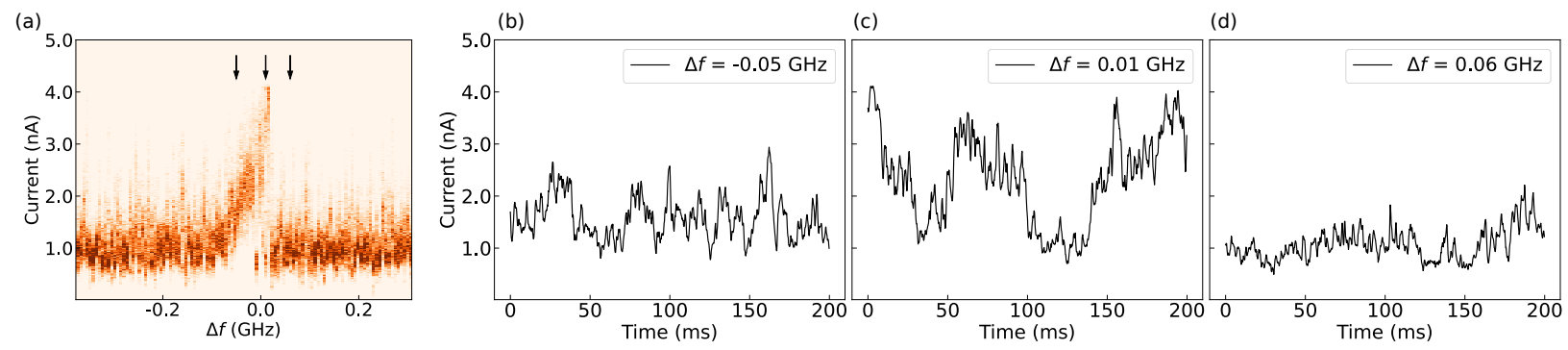

Figure S2: A FinFET device labelled as Er-2 that shows a gradual change of the current. (a) The spectrum of an $\mathrm{Er}^{3+}$ transition with a resonant frequency of 194,205.79 GHz. $\Delta f=$ frequency - 194,205.79 GHz is the laser frequency detuning. (b)-(d) show three current-time traces taken from (a) at different frequency detunings $(\Delta f)$ indicated the three black arrows. 

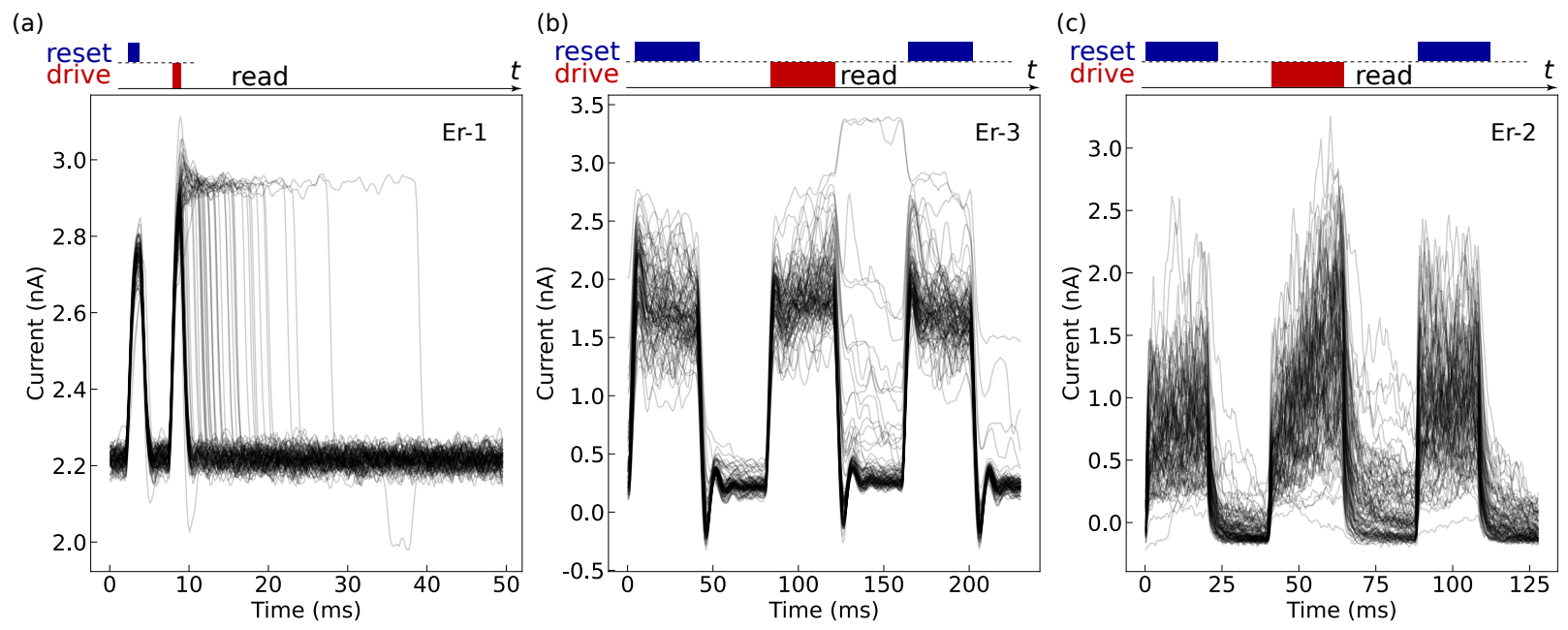

Figure S3: Different types of current signals in three different devices. (a) The current signal during the 'read' phase in device Er-1 shows two discrete current levels. This is plotted with the same data as Fig. 3(a) in the main manuscript. (b) The current signal during the 'read'

phase in another device Er-3 shows multiple discrete levels. (c) The current signal during the 'read' phase in device Er-2 shows gradual variations.

\section{References}

(1) Sellier, H.; Lansbergen, G.; Caro, J.; Rogge, S.; Collaert, N.; Ferain, I.; Jurczak, M.; Biesemans, S. Subthreshold channels at the edges of nanoscale triple-gate silicon transistors. Applied Physics Letters 2007, 90, 073502.

(2) Elzerman, J. M.; Hanson, R.; Willems van Beveren, L. H.; Witkamp, B.; Vandersypen, L. M. K.; Kouwenhoven, L. P. Single-shot read-out of an individual electron spin in a quantum dot. Nature 2004, 430, 431-435.

(3) Yin, C.; Rancic, M.; de Boo, G. G.; Stavrias, N.; McCallum, J. C.; Sellars, M. J.; Rogge, S. Optical addressing of an individual erbium ion in silicon. Nature 2013, 497, 91. 